\title{
A Compact Subsampling Impedance Analyzer for Resonating Sensors
}

Alexander O. Niedermayer, Thomas Voglhuber-Brunnmaier, Erwin K. Reichel, Bernhard Jakoby Institute for Microelectronics and Microsensors

Johannes Kepler University

Altenbergerstr. 69, 4040 Linz, Austria

Phone: +43732 24689301

Fax: +43732 24689316

\section{Introduction}

Resonant sensors are used in a wide range of applications, e.g. as microbalances, chemical sensors in liquid and gaseous environments, and for physical property sensing of liquid and viscoelastic media [1]. Our sensor electronics [2] will be used for the readout of resonant sensors for liquid properties, which means that the Q-factor of such devices is much lower than that of resonators operating in vacuum or gaseous environments. Hence oscillator circuits are not preferable evaluation circuits for this application. Moreover, the damping may be frequency dependent, so measurement over a wide frequency range is mandatory. Besides the common method of using precise laboratory instruments for the measurement of the impedance spectra of these sensors, various approaches have been reported [3-8]. For the interpretation of the measurement results not only the amplitude but also the phase angle of the sensor impedance is of interest allowing a more accurate characterization of the resonance behavior. In this paper we introduce the system concept and some measurement results in comparison to results obtained with a commercial lock-in amplifier.

\section{Measurement Concept}

Exposing a thickness shear mode quartz crystal resonator ( $Q C R)$ to a liquid changes its resonance frequency and the damping of the oscillation as well. To accommodate this effect, the equivalent circuit can be adapted as shown in Fig. 1. The elements of the unperturbed QCR are supplemented with $L_{\mathrm{L}}$ and $R_{\mathrm{L}}$ representing the shift in resonance frequency and the damping, both being influenced by viscosity and density of the medium. Liquid conductivity furthermore results in a spurious (typically nonlinear) shunt admittance $G_{\mathrm{S}}$.
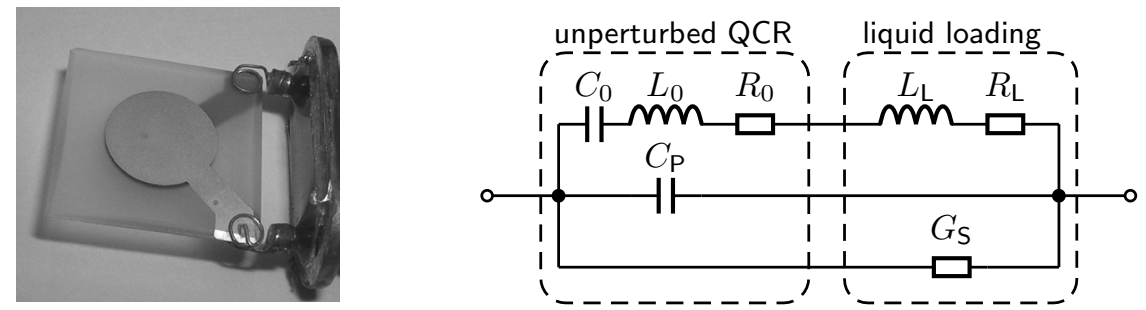

Figure 1: A conventional thickness shear mode QCR and the corresponding equivalent network when exposed to a liquid.

The designed interface electronics was primarily dedicated to monitor the viscosity during a Zeolite synthesis process coming with harsh chemical environment of conductive liquid at a pH-value up to 13 [10]. To avoid negative effects due to $G_{\mathrm{S}}$, in this application only one side of the sensor is in contact with the liquid as shown in Fig. 2. Additionally, the immersed electrode has to be grounded to eliminate any possibility of interference with other sensors used for monitoring the process.

\section{Circuit Overview}

The basic idea of the presented design was to avoid analog modulation components in the signal path in order to increase the accuracy of the measurement results. The sensor interface must be able to handle grounded resonators requiring either a floating current measurement or a current driven excitation of the QCR. Using a signal source based on direct digital sythesis (DDS) not only offers minimal harmonic spurs but also gives the ability of determining the frequency of the signal and thus avoiding the need of an accurate frequency counter. 
Samplechamber
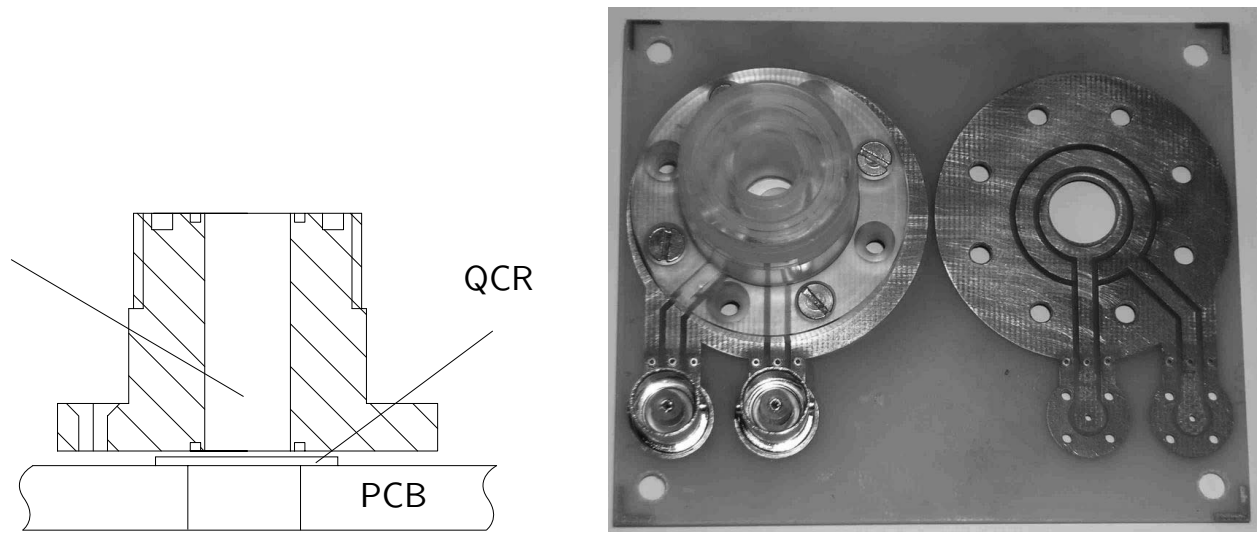

Figure 2: Experimental sensor setup. Only one electrode of the QCR is contacted by the liquid. Although one electrode is grounded in the desired application, the sensor features connectors for both electrodes to provide the opportunity of reference measurements using other evaluation circuits [9].

The used device offers two channels with independent frequency, phase, and gain based on a common system clock providing the option of synchronizing them in a well defined way.

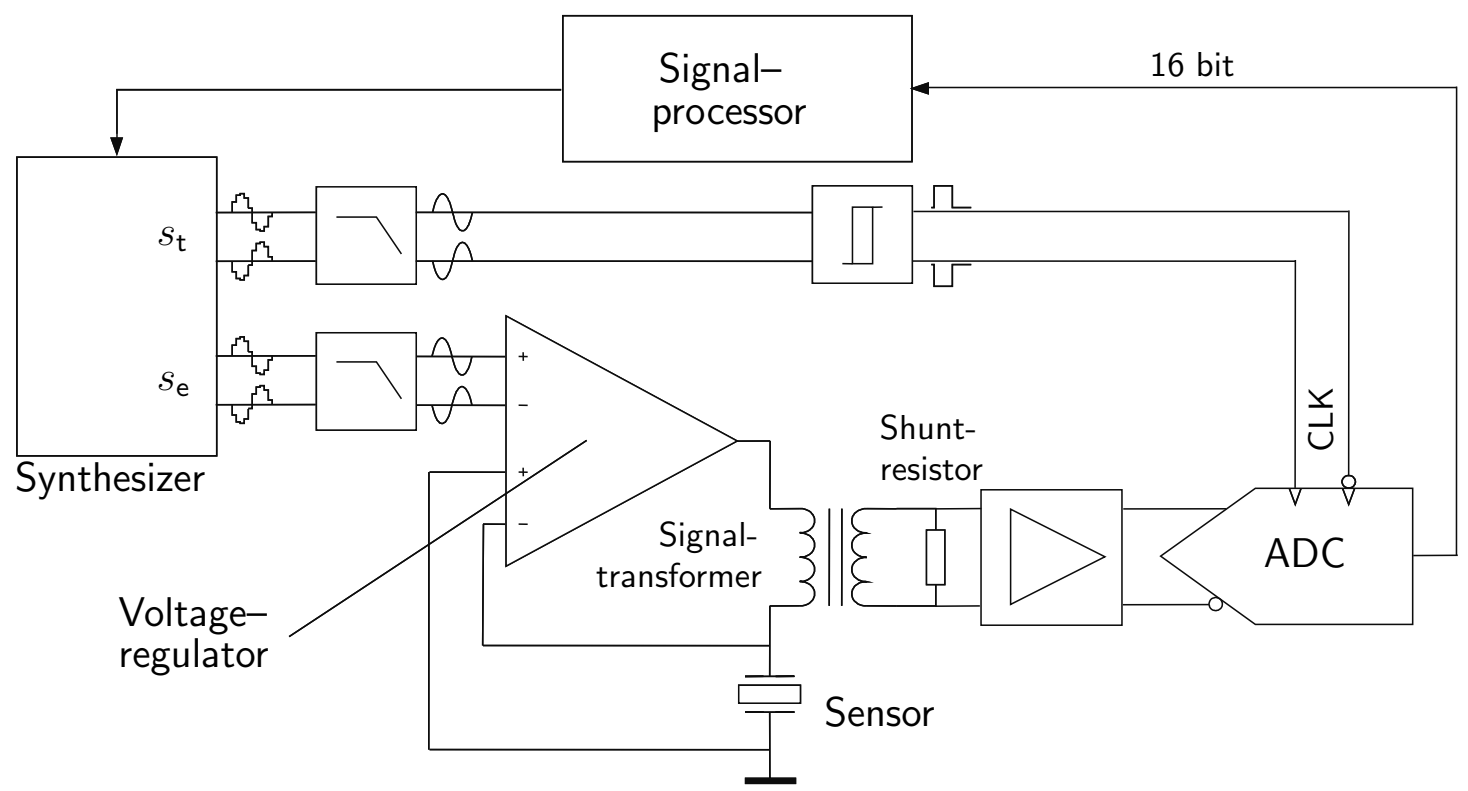

Figure 3: Overview of the impedance analyzer circuit. A signal transformer removes the common mode component from the current signal. Signal and clock paths are routed as differential pairs. The ADC is triggered via a high-speed very low jitter comparator.

As depicted in Fig. 3, a DDS-synthesizer (AD9958) generates two sinusoidal signals, an excitation signal

$$
s_{\mathrm{e}}(t)=a \cdot \sin \left(2 \pi f_{\mathrm{e}} t\right)
$$

with tunable amplitude $a$ for driving the sensor and a trigger signal

$$
s_{\mathrm{t}}(t)=\sin \left(2 \pi f_{\mathrm{t}} t\right)
$$

for clocking the ADC.

The excitation signal $s_{\mathrm{e}}$ is applied to the quartz resonator via the sensor voltage regulator consisting of a high speed differential receiver amplifier (AD8130). This specific device offers conversion to a single-ended signal and amplification in one single stage reducing circuit complexity and avoiding spurs and noise from additional active components. The sensor current is coupled out through a wideband signal transformer eliminating the common mode component of the measured shunt voltage. Hence a higher resolution due to a smaller measurement range 
is achieved when digitizing the sensor current. The galvanic separation additionally provides another positive effect: Usually the analog and digital ground planes on the PCB board have to be connected at mixed signal components. This would result in a ground loop from the DDS to the ADC in the presented design. The signal transformer breaks this loop yielding better noise performance.

The voltage taken from the shunt resistor, is amplified using a low distortion fully differential ADC driver (AD8352) and coupled to the selected differential 16 bit AD converter (AD9460). The symmetric clock inputs of the converter are driven by a very fast SiGe comparator (ADCMP572) deriving the trigger clock from the sinusoidal output of the lowpass filter offering a trigger clock with minimal aperture jitter.

As the clock source of the digital signal processor (DSP) is not synchronized to the data rate of the ADC, the data has to be buffered in an asynchronous FIFO-buffer. The used DSP (ADSP21262) offers an eight stage asynchronous input FIFO with direct memory access at a maximum data rate of 50 MSPS which makes this device quite suitable for the desired application.

When performing the (de)modulation by a DSP, accurate sampling is of particular importance. Even when reducing the sampling rate by subsampling, the analog bandwidth of the system has to exceed the signal frequency. Subsampling is an appropriate way to capture small bandwidth signals and, as the system is excited at one discrete frequency, this condition is fulfilled inherently.

For determining the complex impedance of the resonator usually two components (voltage and current) of the signal have to be recorded. Exciting the sensor with a specified voltage signal provides the option of measuring only one component in amplitude and phase, so there is no need for a second ADC or the implementation of a switch in the signal path. The phase relation therefore is determined by coupling the sampling instance of the $A D C$ and the DDS reducing the remaining clocking uncertainty to a time jitter. As only one signal has to be processed and no frequency estimation is required, the signal processing effort is also reduced by coupling ADC clock and excitation signal.

\section{Signal Processing}

In the proposed configuration, the shunt voltage is (de)modulated by the ADC clock, yielding a signal

$$
\begin{aligned}
& s_{\mathrm{s}}[k]=a \cdot \sin \left(2 \pi f_{\mathrm{s}} k T\right) \quad \mid \quad f_{\mathrm{s}}=f_{\mathrm{e}} \pm f_{\mathrm{t}} \\
& T=\frac{1}{f_{\mathrm{t}}}
\end{aligned}
$$

which is a subsampled replication of $s_{\mathrm{e}}$.

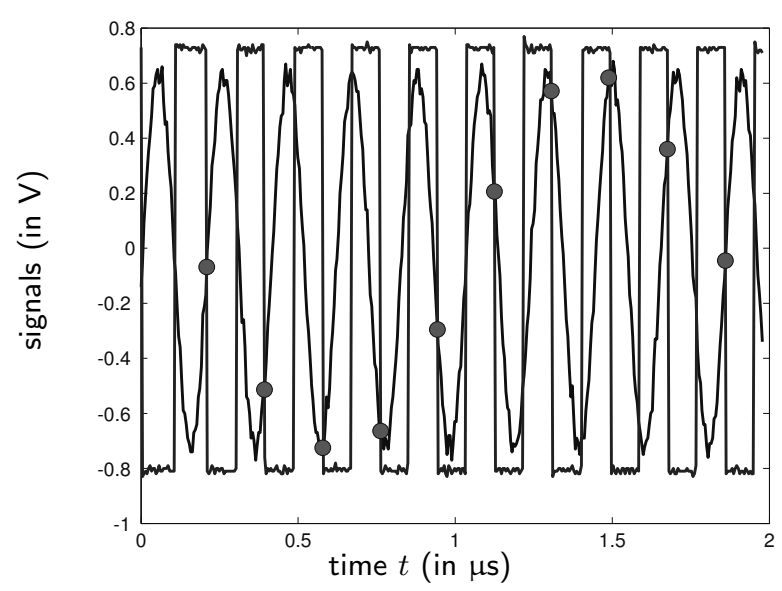

Figure 4: Shunt voltage and trigger signal during an acquisition with $n=9$ samples per $m=1$ period. The depicted signals were acquired without differential probe and hence show only one component of the differential signal including common mode noise.

To obtain a scan of $n$ samples per $m$ periods of $s_{\mathrm{s}}$ (in the subsampled domain), the trigger frequency $f_{\mathrm{t}}$ can be derived from a given excitation frequency $f_{\mathrm{e}}$ by

$$
f_{\mathrm{t}}=f_{\mathrm{e}} \cdot \frac{n}{n-m}
$$


A reference phase angle is preassigned to zero by synchronously resetting the phase accumulators of both DDS channels.

Actually, for the determination of the real and imaginary component of the sensor current it would be reasonable to sample the signal amplitude at two different phase angles (setting $\frac{n}{m}=2$ ). Averaging a number of samples taken at the same phase angle would be the straight forward approach in increasing the signal to noise ratio. Considering different noise sources, this approach only reduces uncorrelated noise but does not have an impact on correlated noise like harmonic distortions or interferences. Taking samples at many different phase angles, as suggested in Fig. 4, enables the treatment of deterministic noise by appropriate signal processing algorithms. So increasing phase resolution has to be preferred to ordinary averaging.

The determination of the complex impedance of the sampled scan is performed by the DSP using Goertzel's algorithm [11], which is known to be the fastest implementation. In order to compensate the different group delay of signal and trigger path and cable latency, the circuit has to be calibrated using a well known (e.g. purely resistive) load.

\section{Results}

In Fig. 5 results obtained with the presented prototype are compared to results from common laboratory equipment (Stanford Research SR844 lock-in amplifier). The experiment was conducted using a one-sided sensor setup as shown in Fig. 2 operating in isopropanol and air with an AT-cut QCR of $12 \mathrm{~mm}$ diameter and a resonance frequency about $4,89 \mathrm{MHz}$. For each of the 300 frequency steps, $n=200$ samples were taken after a decaytime of 20 ms to minimize disturbance by transient oscillations. Including data transfers and control procedure overhead the experiment took about $10 \mathrm{~s}$ per frequency response for both, the lock-in amplifier as well as the impedance analyzer.
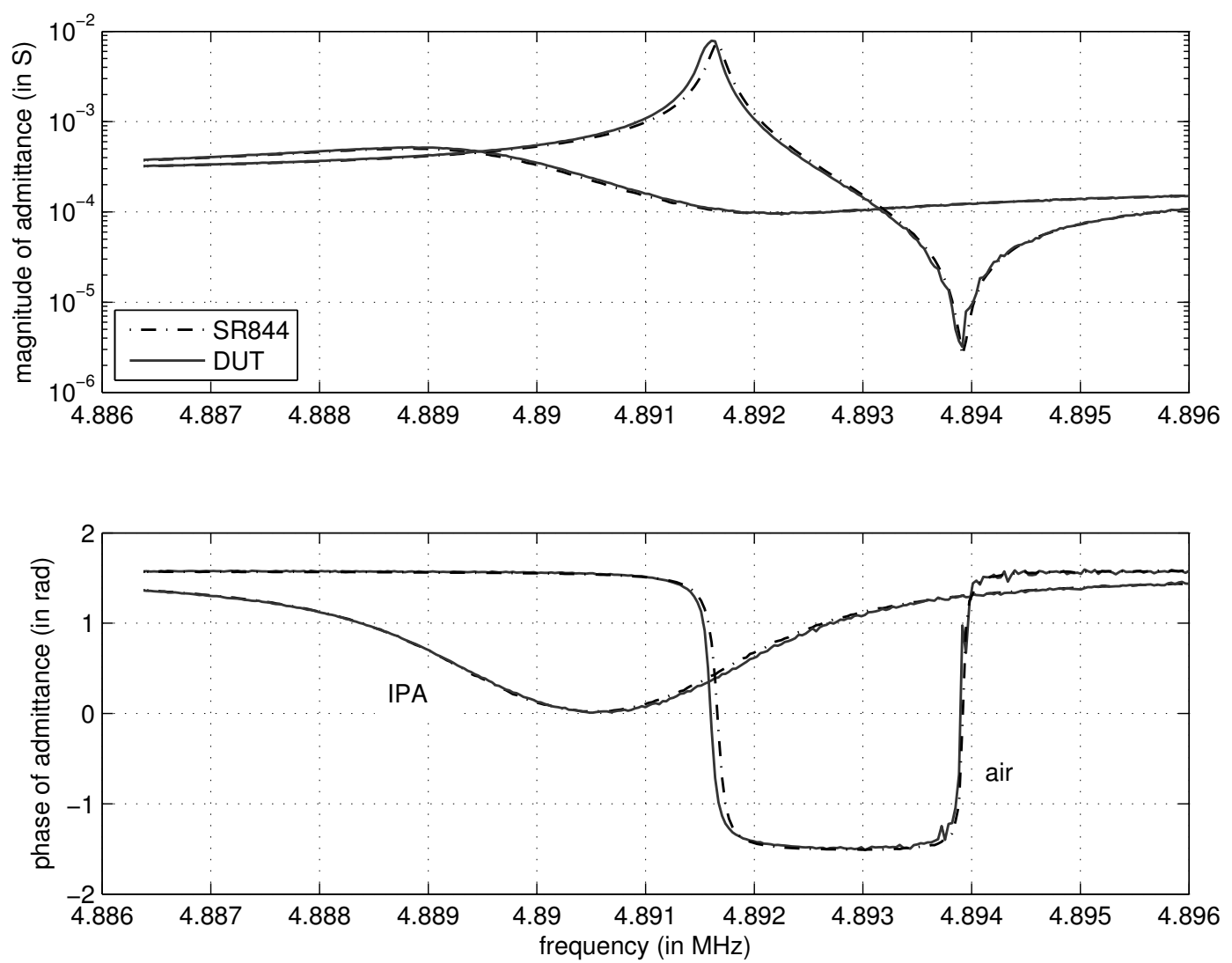

Figure 5: Impedance spectrum of a QCR resonating in air and isopropanol (IPA) acquired with a lock-in amplifier (Stanford Research, SR844) and the presented prototype (device under test, DUT). Circuits, cable latencies and parallel capacitance were calibrated using a reference load (50 $\Omega$ terminating resistor). 


\section{Conclusions}

The presented method to evaluate the frequency response of a quartz crystal sensor promises high precision at low signal processing effort. The number of (active) components in the signal path is kept as low as possible, and no analog (de)modulation is required. To omit the sampling of a second signal such as the resonator voltage in this contribution, two requirements have to be fulfilled. First, the excitation signal has to be determined a priori, which is done by the digital amplitude scaling of the DDS output. Second, the phase relation of voltage and current is evaluated by the coupling of ADC-clock and DDS in a definite way.

With respect to aperture jitter noise, clock generation using DDS is an ideal choice anyway.

Furthermore the presented design concept is rather flexible and provides the opportunity of adapting the tradeoff between speed and resolution.

\section{Acknowledgments}

This work was supported by the Austrian Science Foundation FFG (Project SERMER).

\section{References}

[1] P. Hauptmann, Acoustic Microsensors - State-of-the-Art and Applications, Proc. SENSOR Conference, Nürnberg, 2007, II, 9-14.

[2] A.O. Niedermayer, E.K. Reichel, B. Jakoby, Yet Another Precision Impedance Analyzer (YAPIA) for Resonating Sensors, Proc. EUROSENSORS XXII, Dresden, 2008

[3] F. Eichelbaum, R. Borngräber, J. Schröder, R. Lucklum, P. Hauptmann, Interface circuits for quartz-crystalmicrobalance sensors, Review of Scientific Instruments 70, May 1999, 2537-2545

[4] J. Schröder, R. Borngräber, R. Lucklum, P. Hauptmann, Network analysis based interface electronics for quartz crystal microbalance, Review of Scientific Instruments 72, June 2001, 2750-2755

[5] R. Schnitzer, Development of a microprocessor controlled multi-channel measurement system for resonant piezoelectric sensors, PhD thesis, Vienna, 2006

[6] C. Riesch, B. Jakoby, Novel Readout Electronics for Thickness Shear-Mode Liquid Sensors Compensating for Spurious Conductivity and Capacitances, IEEE Sens. J. 7, 2007, 464-469.

[7] M. Ferrari, V. Ferrari, K. Kanazawa, Dual-harmonic oscillator for quartz crystal resonator sensors, Sens. Act. A $145-146,2008,131-138$

[8] S. Doerner, T. Schneider, J. Schröder, P. Hauptmann, Universal Impedance Spectrum Analyzer for Sensor Application, Proc. IEEE Sensors, Toronto, 2003, 596-599

[9] E. Reichel C. Riesch, W. Hilber, L. Follens, C. Kirschhock, B. Jakoby, Optimized Design of Quartz Disc Viscosity Sensors for the Application in Harsh Chemical Environments, Proc. International Congress on Ultrasonics, Vienna, 2007

[10] L.R.A Follens, E.K. Reichel, C. Riesch, J. Vermant, J.A. Martens, C.E.A. Kirschhock, B. Jakoby, Viscosity sensing in heated alkaline zeolite synthesis media, accepted for Phys. Chem. Chem. Phys. 2008

[11] Alan V. Oppenheim, Ronald W. Schafer, Discrete-time Signal Processing, Prentice Hall, 1998 